\title{
A hybrid method using FAHP and TOPSIS for project selection
}

\author{
Xuan $\mathrm{Li}^{\mathrm{a}}$, Jiang Jiang ${ }^{\mathrm{b}}$ and Su Deng ${ }^{\mathrm{c}}$
}

College of Information System and Management, National University of Defense Technology,

Changsha 410073, China

axlhunan@126.com, jijiangjiangnudt@163.com, bsudeng@nudt.edu.cn

Keywords: project selection, FAHP, TOPSIS.

\begin{abstract}
Like any other forms of decision analysis that involve multiple criteria, project selection is an unstructured, complex decision problem with lots of aspects that must be taken into consideration. A hybrid method combining fuzzy analytic hierarchy process (FAHP) and the technique for preference by similarity to ideal solution (TOPSIS) is proposed to solve this problem. FAHP is used to analyze the problem and meanwhile to determine the criteria weights. TOPSIS is applied to rank all the given alternatives and determine the appropriate one. A case is studied to demonstrate the usefulness and effectiveness of the hybrid method.
\end{abstract}

\section{Introduction}

Project selection, one of the most common issues in managerial decision making, can exert a great influence on organizations [1]. The importance of proper project selection cannot be over-emphasized. However, like any other forms of decision analysis, selecting the appropriate project among a given set of alternatives is a multi-criteria decision making (MCDM) problem, wherein lots of data as well as conflicting criteria need to be handled. During the past decade, many methods have been proposed to solve MCDM problems, one of which includes analytic hierarchy process (AHP) [2]. It is proposed with the aim at quantifying relative priorities on a ratio scale for a given set of alternatives, by strictly agreeing with consistency in the decision-making process. However, it may be uneasy for decision makers to assign crisp values to the comparison judgments in the decision-making process in many situations, in which cases fuzzy AHP (FAHP) is used. It has been widely used to assign weights in different applications [3-4].

The technique for preference by similarity to ideal solution (TOPSIS) method [5] was developed for multi-criteria optimization of complex systems. It determines the best alternative that is closest to the positive-ideal solution (PIS) and meanwhile farthest from the negative-ideal solution (NIS). This method has been applied in different kinds of selection problems. Taylan et al. [3] combined fuzzy AHP and fuzzy TOPSIS methodologies to construction projects selection and risk assessment. Aloini et al. [6] proposed a peer-based modification to intuitionistic fuzzy multi-criteria group decision making with TOPSIS method for packaging machine selection. Beikkhakhian et al. [7] proposed an ISM model based on TOPSIS-AHP methods in evaluating agile suppliers selection criteria and ranking suppliers.

In this paper, a hybrid method based on FAHP and TOPSIS is proposed for project selection. FAHP is used to determine the criteria weights, which is good at coping with the vagueness and uncertainty. TOPSIS is then used to rank the solutions combined with the criteria weights. The proposed method can better cope with the vagueness associated with information, and help decision makers choose among the alternatives.

\section{Analytical Methods}

In this Section, we focused mainly on some basic definitions related with fuzzy set theory, FAHP and TOPSIS, used in the proposed method. 
Fuzzy set theory. Fuzzy set theory was first introduced by Zadeh [8] to deal with possible vague ambiguity and uncertainty in most real life situations. Some basic definitions related with fuzzy sets are given as follows:

Definition 1. Fuzzy set $\tilde{A}$ is defined as a set of organized pairs:

$$
\tilde{A}=\left\{\mathrm{x}, \mu_{\tilde{A}}(\mathrm{x}) \mid x \in X\right\},
$$

where fuzzy set $\tilde{A}$ is defined on the universe set $X \in R$. In general, set $X$ can be either finite or infinite, $\mu_{\tilde{A}}(\mathrm{x})$ is a membership function of fuzzy set $\tilde{A}$ [8].

Definition 2. A triangular fuzzy number (TFN) can be expressed as a triplet $(a, b, c)$. Without loss of generality, a TFN $\tilde{a}=(a, b, c)$ is defined by the following membership function [8]:

$$
y=\left\{\begin{array}{cc}
0, & x \leq a, \\
\frac{x-a}{b-a}, & a<x \leq b, \\
\frac{c-x}{c-b}, & b<x \leq c, \\
0, & x>c,
\end{array}\right.
$$

Definition 3. The $\alpha$-cut of the fuzzy set $\tilde{A}$ of the universe of discourse $X$ is defined as follows:

$$
A_{\alpha}=\left\{x \in X \mid u_{\tilde{A}}(x) \geq \alpha\right\} \text { where } \alpha \in[0,1]
$$

Definition 4 . The TFN can be characterized by defining the interval of confidence level $\alpha$ using (4).

$$
\forall \alpha[0,1] \tilde{\mathrm{M}}_{\alpha}=\left[l^{\alpha}, u^{\alpha}\right]=[(m-l) \alpha+l,-(u-m) \alpha+u]
$$

Definition 5. Let $\tilde{a}_{1}=\left(a_{1}, b_{1}, c_{1}\right)$ and $\tilde{a}_{2}=\left(a_{2}, b_{2}, c_{2}\right)$ be two TFNs, then the vertex method is defined to calculate the distance between them:

$$
d\left(\tilde{a}_{1}, \tilde{a}_{2}\right)=\sqrt{1 / 3\left[\left(a_{1}-a_{2}\right)^{2}+\left(b_{1}-b_{2}\right)^{2}+\left(c_{1}-c_{2}\right)^{2}\right]}
$$

FAHP. AHP, introduced by Saaty [2], is good at coping with tangible and intangible factors in a systematic way, and providing a structured yet relatively simple solution to the decision making problems. Yet its main limitation lies in handling the uncertainty and ambiguity in real-life. Therefore, FAHP extends AHP by combining it with fuzzy set theory to tackle the ambiguities involved. The FAHP method can be described into a few steps as follows:

Step 1: Initially, the complex problem needs to be decomposed into a hierarchical structure. In general, it is decomposed into a hierarchy of goal, criteria and alternatives. It can also be expanded if necessary, according to the specific problems.

Step 2: The second step is the comparison of the alternatives and the criteria. In this step, prioritization procedure can be done in order to determine the relative importance of the criteria. In this study, five TFNs $(\tilde{1}, \tilde{3}, \tilde{5}, \tilde{7}, \tilde{9})$, whose linguistic variables and membership functions are "equally important $(1,1,3)$ ”, “weakly important $(1,3,5)$ ”, “strongly more important $(3,5,7)$ ” , "very strongly important $(5,7,9)$ ” and "extremely more important $(7,9,11)$ ", respectively.

The result of the pairwise comparison on $n$ criteria can be summarized in fuzzy comparison matrix $\tilde{A}$, where $\tilde{a}_{i j}=1$ if $i$ equals to $j$ and $\tilde{a}_{i j}=(\tilde{1}, \tilde{3}, \tilde{5}, \tilde{7}, \tilde{9})$ or $\tilde{1}^{-1}, \tilde{3}^{-1}, \tilde{5}^{-1}, \tilde{7}^{-1}, \tilde{9}^{-1}$ if $i$ differs with $j$.

Step 3: Converting the fuzzy comparison matrix into a crisp comparison matrix

Adamo [9] proposed $\alpha$-cut method to rank the fuzzy numbers. While $\alpha$ is determined, the comparison matrix can be turned using (4). Then the degree of satisfaction can be estimated by the index of optimism $\mu$ [10]. The lager value of index $\mu$, the higher degree of optimism. Then, the fuzzy comparison matrix $\tilde{A}$ is converted into crisp comparison matrix $A$ by using (6).

$$
\tilde{a}_{i j}^{\alpha}=\mu a_{i j u}^{\alpha}+(1-\mu) a_{i j l}^{\alpha} \quad 0<\mu \leq 1
$$

Step 4: A consistency test is needed to testify the reasonability of the decision makers' pairwise comparison. To calculate consistency ratio (CR), the consistency index is needed, which could be calculated with (7). 


$$
\text { C.I. }=\frac{\lambda_{\max }-n}{n-1}
$$

where $\lambda_{\max }$ is the largest eigenvalue of the pair wise comparison matrix, $n$ is the matrix order, and RI is random index.

$$
\begin{aligned}
& A_{w}=\lambda_{\max } w \\
& \text { C.R. }=\frac{\text { C.I. }}{\text { R.I. }}
\end{aligned}
$$

The number 0.1 is the accepted upper limit for CR. If the final consistency ratio exceeds this value, the evaluation procedure has to be repeated until desired consistency is reached.

TOPSIS. The TOPSIS method is an applicable MCDM method, which can help the decision makers rank the solutions with conflicting criteria and reach a final solution. This ranking method focuses on a set of finite alternatives that are evaluated according to each criterion function. The main steps of the TOPSIS method are described in depth as:

Step 1: Calculate the normalized quantities by using (9).

Suppose that there are altogether $m$ alternatives and $n$ criteria. Then, the normalized decision matrix $R=\left(r_{i j}\right)_{m \times n}$ can be obtained.

$$
r_{i j}=\frac{x_{i j}}{\sqrt{\sum_{i=1}^{m} x_{i j}^{2}}}, i=1,2, \ldots, m ; \mathrm{j}=1,2, \ldots, n,
$$

where $x_{i j}$ is the characteristic data of alternative $i$ on criterion $j$.

Step 2: Calculate the weighted normalized decision matrix $\tilde{R}=\left(v_{i j}\right)_{m \times n}$ by using the following relation:

$$
v_{i j}=w_{j} \times r_{i j},
$$

where $w_{j}$ is the weight of the $j$ th criterion, determined by FAHP.

Step 3: Determine the PIS $v_{j}^{*}$ and the NIS $v_{j}^{-}$values of all criteria ratings.

$$
\begin{aligned}
& v_{j}^{*}=\left\{\begin{array}{c}
\max _{i} v_{i j}, \text { for benefit criteria } \\
\min _{i} v_{i j}, \text { for cost criteria }
\end{array}\right\}, i=1,2, \ldots, m, \\
& v_{j}^{-}=\left\{\begin{array}{c}
\min _{i} v_{i j}, \text { for benefit criteria } \\
\max _{i} v_{i j}, \text { for cost criteria }
\end{array}\right\}, i=1,2, \ldots, m .
\end{aligned}
$$

Step 4: Calculate the relative distance of each alternative from PIS, given as follows:

$$
D_{i}^{+}=\sqrt{\sum_{j=1}^{n}\left(v_{i j}-v_{j}^{*}\right)^{2}} i=1,2, \ldots, m
$$

Similarly, the relative distance of each alternative from NIS is calculated as:

$$
D_{i}^{-}=\sqrt{\sum_{j=1}^{n}\left(v_{i j}-v_{j}^{-}\right)^{2}} i=1,2, \ldots, m
$$

Step 5: Calculate the coefficient of each solution using:

$$
C C_{i}=\frac{D_{i}^{-}}{D_{i}^{+}+D_{i}^{-}}, i=1,2, \ldots m
$$

Finally, the ranking order of the given set of alternatives can be obtained based on the $C C$ values. The larger the index value, the better performance of the corresponding alternative. 


\section{Proposed Method}

The proposed approach for project selection, composed of FAHP and TOPSIS methods, consists of several steps. As is shown in Fig. 1, it displays an overview of the proposed FAHP-TOPSIS hybrid method. In the first step, the problem needs to be broken down into a hierarchy of interrelated decision elements, including the goal, criteria, and the given set of alternatives. Next, characteristic data of the criteria should be collected, which will be used in the next step. Subsequently, it aims at generating the weights of the criteria by FAHP. Noted that when applying FAHP to decide the weights, the decision group uses their knowledge and experience to form the pairwise comparison on $n$ criteria. The results can be summarized in a fuzzy evaluation matrix. Then, weights of the criteria are determined by using eigenvector method. It's necessary to measure whether or not inconsistency occurs in the judgment process at all levels. If not, decision makers should revise the original values in the pair wise comparison matrix until desired consistency level reached. The criteria weights are then inputted into a TOPSIS model to rank the given set of alternatives.

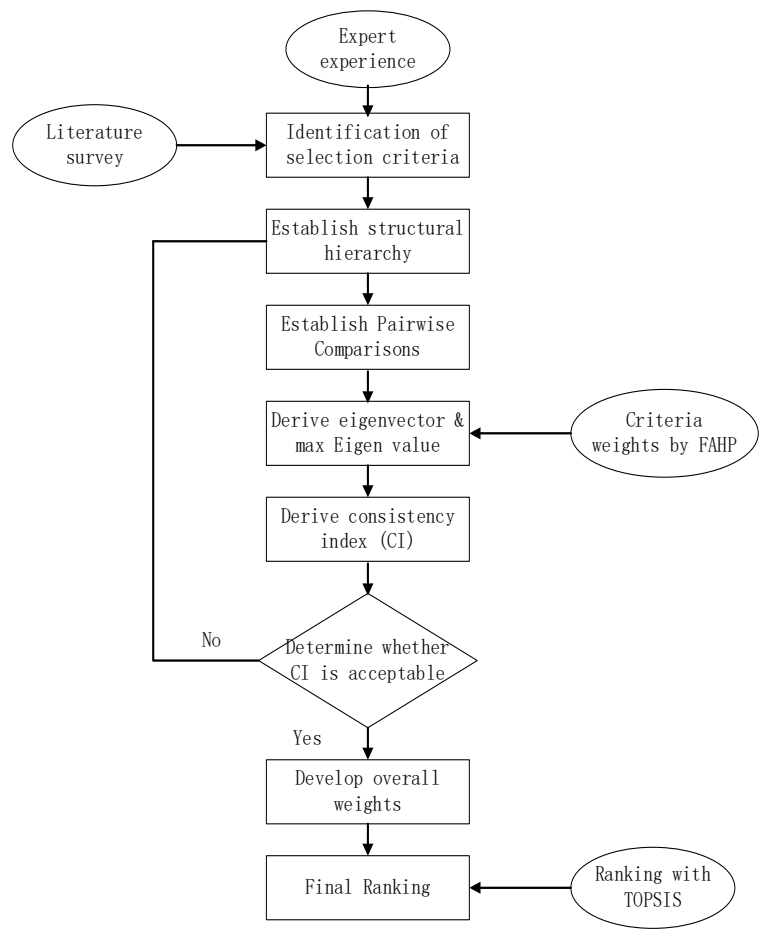

Fig. 1 Hybrid method procedure

\section{Case study}

To fully illustrate the utilization of the proposed hybrid method, a case is studied on project selection.

Problem setup and data. As aforementioned, a three-level hierarchical structure is first set up, including the final goal, the criteria, and the alternatives. To this aim, a survey is conducted to select the appropriate criteria. Suppose after careful discussion, five criteria are finally determined for the project selection problem, denotes as $C_{1}, C_{2}, C_{3}, C_{4}$, and $C_{5}$, respectively.

After the identification of these five criteria for project selection, the expert team was asked to conduct a pairwise comparison with regard to different criteria in the next stage by using linguistic variables mentioned above. After careful discussion and study, the final matrices are shown in Table 1 as follows: 
Table 1 Pairwise comparision matrix of the major criteria

\begin{tabular}{cccccc}
\hline Criteria & $C_{1}$ & $C_{2}$ & $C_{3}$ & $C_{4}$ & $C_{5}$ \\
\hline$C_{1}$ & 1 & $\tilde{3}$ & $\tilde{9}$ & $\tilde{5}$ & $\tilde{9}$ \\
$C_{2}$ & $\tilde{3}^{-1}$ & 1 & $\tilde{5}$ & $\tilde{3}$ & $\tilde{9}$ \\
$C_{3}$ & $\tilde{9}^{-1}$ & $\tilde{5}^{-1}$ & 1 & $\tilde{3}^{-1}$ & $\tilde{3}$ \\
$C_{4}$ & $\tilde{5}^{-1}$ & $\tilde{3}^{-1}$ & $\tilde{3}$ & 1 & $\tilde{7}$ \\
$C_{5}$ & $\tilde{9}^{-1}$ & $\tilde{9}^{-1}$ & $\tilde{3}^{-1}$ & $\tilde{7}^{-1}$ & 1 \\
\hline
\end{tabular}

The fuzzy comparison judgments of the five criteria related with the overall objective are then obtained. Weights of the criteria are determined by using eigenvector method. It is quite necessary to measure whether or not consistency exists at all levels. In this study, all the CRs were less than 0.1 and consequently the matrices were found to be reasonable.

TOPSIS. The characteristic data about the project alternatives relating to the criteria are collected and shown in Table 2.

Table 2 Characteristic data on alternative projects

\begin{tabular}{cccccc}
\hline & \multicolumn{5}{c}{ Criteria } \\
\cline { 2 - 6 } Alternatives & $C_{1}$ & $C_{2}$ & $C_{3}$ & $C_{4}$ & $C_{5}$ \\
& 0.5033 & 0.2635 & 0.0611 & 0.1409 & 0.0311 \\
\hline System 1 & 8 & 8.5 & 7.5 & 180 & 8 \\
System 2 & 6 & 6 & 6 & 100 & 5 \\
System 3 & 9 & 9 & 8 & 220 & 9 \\
System 4 & 7 & 8 & 7 & 160 & 7 \\
System 5 & 8 & 6.5 & 8 & 170 & 6 \\
System 6 & 9 & 7.5 & 8 & 200 & 9 \\
\hline
\end{tabular}

The TOPSIS method is then used for the ranking of all the alternatives. The obtained data are first normalized using Eq. (9), and the weighted normalized decision matrix can be obtained using Eq. (10).

Table 3 Results of alternatives with TOPSIS

\begin{tabular}{cllllll}
\hline Alternatives & System 1 & System 2 & System 3 & System 4 & System 5 & System 6 \\
\hline$D_{i}{ }^{+}$ & 2.578 & 7.0496 & 2.6701 & 3.7648 & 5.7015 & 2.4547 \\
$D_{i}{ }^{-}$ & 5.3505 & 2.6701 & 7.0496 & 3.7769 & 1.9517 & 7.0377 \\
$C$ & 0.6748 & 0.2747 & 0.7253 & 0.5008 & 0.2550 & 0.7414
\end{tabular}

Then, the relative distance $D^{+}$and $D^{-}$of each alternative from the PIS and NIS were obtained using Eqs. (12) and (13). The closeness coefficient for each alternative is obtained by Eq. (14). Finally, all the given set of alternatives can be ranked by comparing the $C C_{i}$ values, shown in Table 3 . Therefore, we can find that the ranking of the alternatives in descending order are as follows:

system $6>$ system $3>$ system $1>$ system $4>$ system $2>$ system 5 .

Thus, we can come to a conclusion that system 6 is selected as the appropriate one among all the six alternatives with the maximum closeness. 


\section{Conclusions}

The project selection is a complex MCDM problem. In general, lots of different aspects need to be taken into consideration. Meanwhile, it commonly occurs in real life situations that experts may experience difficulty assigning crisp values to criteria or alternatives. Thus, a hybrid method combined with FAHP and TOPSIS is proposed. FAHP is good at coping with the uncertainties associated with information, and is used to calculate the evaluation criteria weights. Then, the weights are inputted in the TOPSIS model to rank the alternatives. The empirical case is presented to demonstrate the application of the proposed approach. The proposed approach can also be used in various MCDM problems and some other techniques can be combined to improve the proposed approach, which is one of the main research directions in our future work.

\section{References}

[1] K. Salehi, A hybrid fuzzy MCDM approach for project selection problem, Decision Science Letters. 4 (2015) 109-116.

[2] T.L. Saaty, The Analytic Hierarchy Process, RWS Publications, PA: Pittsburgh, 1990.

[3] O. Taylan, A.O. Bafail, R.M.S. Abdulaal, et. al, Construction projects selection and risk assessment by fuzzy AHP and fuzzy TOPSIS methodologies, Applied Soft Computing. 17 (2014) 105-116.

[4] H.C. Liu, J.X.You, X.Y. You, et al, A novel approach for failure mode and effects analysis using combination weighting and fuzzy VIKOR method, Applied Soft Computing. 17 (2014) 105-116.

[5] S. Opricovic and G.H. Tzeng, Compromise solution by MCDM methods: a comparative analysis of VIKOR and TOPSIS, European Journal of Operational Research. 156 (2004) 445-455.

[6] D. Aloini, R. Dulmin and V. Mininno, A peer IF-TOPSIS based decision support system for packaging machine selection, Expert Systems with Application. 41(2014) 2157-5165.

[7] Y. Beikkhakhian, M. Javanmardi, M. Karbasian, et al. The application of ISM model in evaluating agile suppliers selection criteria and ranking suppliers using fuzzy TOPSIS-AHP methods, Expert Systems with Applications. 42 (2015) 6224-6236.

[8] L.A. Zadeh, Fuzzy sets, Information Sciences. 8 (1965) 338-353.

[9] J.M. Adamo, Fuzzy decision trees, Fuzzy Sets and Systems. 4 (1980) 207-220.

[10] M. Lee, H. Pham and X. Zhang, A methodology for priority setting with application to software development process, European Journal of Operation Research. 118 (1999) 375-389. 